\title{
DYNAMICAL BEHAVIOR OF TRAINS EXCITED BY A NON-GAUSSIAN VECTOR VALUED RANDOM FIELD.
}

\author{
G. Perrin ${ }^{1,2,3}$, C. Soize ${ }^{1}$, D. Duhamel ${ }^{2}$, and C. Funfschilling ${ }^{3}$ \\ ${ }^{1}$ Université Paris-Est, Modélisation et Simulation Multi-Échelle (MSME UMR 8208 CNRS). \\ 5 Bd. Descartes, 77454 Marne-la-Vallée, France. \\ e-mail: guillaume.perrin@enpc.fr, christian.soize@univ-paris-est.fr \\ ${ }^{2}$ Université Paris-Est, Navier (UMR 8205 ENPC-IFSTTAR-CNRS). \\ Ecole Nationale des Ponts et Chaussées, 6 et 8 Avenue Blaise Pascal, Cité Descartes, Champs sur \\ Marne, 77455 Marne-la-Vallée, Cedex 2, France. \\ e-mail: denis.duhamel@enpc.fr \\ ${ }^{3} \mathrm{SNCF}$, Innovation and Research Department. \\ Immeuble Lumière, 40 avenue des Terroirs de France, 75611, Paris, Cedex 12, France. \\ e-mail: christine.funfschilling@sncf.fr
}

Keywords: Karhunen-Loève Reduction, Polynomial Chaos Expansion, Random fields, Railway Track Geometry.

\begin{abstract}
Since 2012, European high speed railway networks are meant to have gone to market. Hence, several high speed trains, such as ICE, TGV, ETR 500, Sapsan..., are likely to run on the same tracks, whereas they have been originally designed for specific and different railway networks. Due to different mechanical properties and structures, the dynamic behaviors, the agressiveness of the vehicle on the track and the probabilities of exceeding security and comfort thresholds will be very different from one train to an other. These maintenance, certification and comfort criteria depend on the dynamic interaction between the vehicle and the railway track and in particularly on the contact loads between the wheels and the rail, which are very hard to evaluate experimentally. Therefore, the numerical simulation is bound to play a key role in this context, as it is able to compute these quantities of interest. Nevertheless, the track-vehicle system being strongly non-linear, this dynamic interaction has to be analyzed not only on a few track portions but on the whole realm of possibilities of running conditions that the train is bound to be confronted to during its lifecycle. In reply to this concern, this paper presents a method to analyze the influence of the track geometry variability on the train behavior, which could be very useful to evaluate and compare the agressiveness of different trains. This method is based on a stochastic modeling of the track geometry, for which parameters have been identified with experimental measurements.
\end{abstract}




\section{INTRODUCTION}

If simulation is introduced in certification and conception processes, it has to be very representative of the physical behaviour of the system. The model has thus to be fully validated and the simulations have to be raised on a realistic and representative set of excitations.

Hence, this work presents a three steps method to characterize the influence of the track geometry on the train dynamics. The first step, which is presented in Section 2, corresponds to the classical description of the studied mechanical problem. A particuler attention has to be paid to the definition of the quantities of interest, as this choice will play a major role on the propagation method. Then, Section 3 deals with the characterization of the input variability. At last, Section 3 presents the propagation of the variability through the mechanical system. Two applications of the method will then be analyzed: the influence of an increase of the speed on the train stability and the quantification of the agressiveness of three high speed trains that have different mechanical properties.

\section{STEP A: DESCRIPTION OF THE STOCHASTIC MECHANICAL MODELING}

This section is devoted to the description of the stochastic modeling of the railway system.

\subsection{Description of the railway dynamic problem}

As presented in Introduction, a railway dynamic problem can be seen as the excitation of the train by the track geometry through the wheel/rail contact forces, where the wheel/rail contact forces are computed from the wheel profile and the rail profile thanks to the Hertz and Kalker theories. The dynamic $\left(u_{i}(t), \dot{u}_{i}(t)\right)$ of each mass body $i$ of the train at each time step $t \geq 0$, that we describe by the vector of the generalized coordinates,

$$
\boldsymbol{U}(t)=\left(u_{1}(t), u_{2}(t), \cdots, \dot{u}_{1}(t), \dot{u}_{2}(t), \cdots\right),
$$

can therefore be determined by solving the Euler-Lagrange equation, which reads:

$$
\frac{d}{d t}\left(\frac{\partial E_{c}}{\partial \dot{u}_{i}}\right)-\frac{\partial E_{c}}{\partial u_{i}}=L_{i}(\boldsymbol{U}, \mathcal{T})
$$

with $E_{c}$ the total kinetic energy of the train, and $L_{i}(\boldsymbol{U}, \mathcal{T})$ the general load that is applied to the mass body $i$, which depends on the track geometry $\mathcal{T}$ and on the generalized coordinates $\boldsymbol{U}$. Eq. (2) can be rewritten in a matricial form as:

$$
[A(\boldsymbol{U})] \dot{\boldsymbol{U}}=\boldsymbol{F}(\boldsymbol{U}, \mathcal{T}),
$$

with $[A]$ and $\boldsymbol{F}$ two strongly non-linear operators. This system is usually solved with an explicit scheme.

The generalized coordinates $\boldsymbol{U}$, for which evolution is computed from Eq. (3), are then post-treated to define the final outputs of the railway simulation. In this work, three criteria are introduced to analyze the vehicule dynamics on a given track geometry of total length $S^{\text {tot }}$ :

- a shifting criterion:

$$
\left(Y_{\ell}+Y_{r}\right)_{\max }=\max _{\text {wheelset }} \max _{w \leq s \leq S^{\text {tot }}}\left\{Y_{\ell}^{w}(s)+Y_{r}^{w}(s)\right\}
$$


- a derailment criterion:

$$
(Y / Q)_{\max }=\max _{\text {wheel }} \max _{q \leq \leq \leq \leq S^{\text {tot }}}\left\{Y_{q}(s) / Q_{q}(s)\right\}
$$

- a wear criterion:

$$
(T \gamma)=\sum_{\text {wheel } q} \int_{0}^{S^{\text {tot }}} T_{q}(s) \gamma_{q}(s) d s
$$

where:

- $Y_{\ell}^{k}$ and $Y_{r}^{k}$ are the left and right lateral forces of the same wheelset $k$, such that the higher $\left(Y_{\ell}+Y_{r}\right)_{\max }$ is, the more chance for a shifting of the track there is;

- $Y_{q}$ and $Q_{q}$ are the lateral and vertical components of the wheel/rail contact force at wheel $q$, such that the higher $(Y / Q)_{\max }$ is, the more on the flange a wheel of the train can be;

- $T_{q}$ and $\gamma_{q}$ are respectively the creep force and the slip at wheel $q$, such that the higher $(T \gamma)$ is, the higher the contact wear is likely to be for one run of the complete train.

Finally, the deterministic railway problem, corresponding to the dynamics of a vehicle $\mathcal{V}$ on a track geometry $\mathcal{T}$ can be expressed as:

$$
(\mathcal{V}, \mathcal{T}) \mapsto \boldsymbol{c}=\boldsymbol{g}(\mathcal{V}, \mathcal{T}), \quad \boldsymbol{c}=\left(\left(Y_{\ell}+Y_{r}\right)_{\max },(Y / Q)_{\max },(T \gamma)\right),
$$

where it is reminded that $\boldsymbol{g}$ is a complex and non-linear operator.

\subsection{Stochastic problem}

In this work, it is supposed that a normalized model of a train is available, for which mechanical parameters are fixed and have been accurately identified. Moreover, two description scales can be distinguished for the track geometry:

- on the first hand, the track design, which corresponds to the parameters of the mean line position, that is to say the vertical curvature $c_{V}$, the horizontal curvature $c_{H}$, and the track superelevation $c_{L}$, is decided once for all at the building of a new track for economical and political reasons;

- on the second hand, for a fixed track design, the track irregularities are in constant evolution, due to the interactions between the train and the track, to the maintenance operations, and to the wheather conditions. There are four kinds of track irregularities: the horizontal and vertical alignment irregularities, $x_{1}$ and $x_{2}$, the cant deficiencies $x_{3}$, and the alignement irregularities $x_{4}$.

It is supposed in this work that the track irregularities characterization can be separated from the track design description. Hence, in the following, a measured track design is chosen and only the track irregularities will be supposed to be variable. In this prospect, let 
$\boldsymbol{X}=\left(X_{1}, X_{2}, X_{3}, X_{4}\right)$ be the vector-valued random field corresponding to the four track irregularities, for which statistical characteristics have to be identified from experimental data. As a consequence, vector $c$, which gathers the three criteria of interest, becomes a random vector $C$, and the stochastic problem can be expressed by:

$$
\boldsymbol{X} \mapsto \boldsymbol{C}=\boldsymbol{G}(\boldsymbol{X}) .
$$

The choice of the quantities of interest is crucial as it will rule the choice of the propagation method. In this work, we are interested in the PDF of each criterion, that are denoted by $p_{C_{i}}$, $1 \leq i \leq 3$.

\section{STEP B: CHARACTERIZATION OF THE INPUT VARIABILITY}

We assume in this work that the track irregularities can be seen as a vector-valued random field, $\boldsymbol{X}=\left(X_{1}, X_{2}, X_{3}, X_{4}\right)$, which gathers the four kinds of track irregularities. Characterizing the input variability of the railway stochastic problem amounts thus to identifying the distribution of $\boldsymbol{X}$ from experimental measurements.

To this end, the measurement train IRIS 320 has been running continuously since 2007 over the French railway network, measuring and recording the track geometry of the main national lines. Based on these experimental measurements, this section aims at developing a methodology to parameterize the physical properties as well as the variability of track irregularities random field $\boldsymbol{X}$. This modeling will allow the numerical generation of track geometries that are physically realistic and statistically representative of a whole railway network.

\subsection{Local-global approach and available information}

In this work, it is supposed that the track irregularities of a whole high speed line of total length $S^{\text {tot }}$ has been measured. The track irregularity vector,

$$
\left\{\boldsymbol{X}(s)=\left(X_{1}(s), X_{2}(s), X_{3}(s), X_{4}(s)\right), s \in\left[0, S^{\text {tot }}\right]\right\},
$$

is a random field with values in $\mathbb{R}^{4}$, for which realizations are continuous functions. It is moreover assumed that $\boldsymbol{X}$ is a centered random field, such that:

$$
E[\boldsymbol{X}(s)]=\mathbf{0}, s \in\left[0, S^{\text {tot }}\right],
$$

with $E[\cdot]$ the mathematical expectation.

Due to the specific interaction between the train and the track, this random field is neither Gaussian nor stationary, which motivates a local-global approach for the characterization of the distribution of $\boldsymbol{X}$. This approach is based on the hypothesis that a whole railway track can be considered as the concatenation of a series of independent track portions of same length $S$, for which physical and statistical properties are the same. Therefore, this asumption should allow us to reduce the complexity of the problem, by restricting the characterization of $\boldsymbol{X}$ to its projection on the interval $\Omega=[0, S]$, with $S \ll S^{\text {tot }}$. Length $S$ plays therefore a key role in the modeling procedure, and its value has to be carefully evaluated. Indeed, $S$ has to be long enough for the statistical and spectral information of $\boldsymbol{X}$ to be accurately taken into account. However, the higher $S$ is, the little number of independent realizations for $\boldsymbol{X}$ can be extracted from the complete measurement of the railway network. 
For confidentiality reasons, length $S$ is not given in this work, and it is assumed that it has been carefully chosen as an optimum of the compromise between computational cost and modeling precision. In the same manner, the spatial quantities will be normalized by length $S$ in the following.

Under the local-global hypothesis, it is now supposed that $\nu^{\exp }$ track portions of same length $S$, that are denoted by $\left\{\boldsymbol{x}^{1}, \cdots, \boldsymbol{x}^{\nu^{\text {exp }}}\right\}$, can be extracted from the experimental measurements. It is reminded that these measurements are supposed to be $\nu^{\exp }$ independent realizations of random field $\boldsymbol{X}$, which defines the maximum available information for the stochastic modeling of the track geometry.

\subsection{Track geometry stochastic modeling}

\subsubsection{Definition of the local model}

The objective of the stochastic modeling is to identify in inverse the statistical properties of $\boldsymbol{X}$ from its $\nu^{\exp }$ independent realizations. This modeling is based on a two steps decomposition. First, a Karhunen-Loève (KL) expansion is performed (see [1, 2, 3, 4, 5, 6, 7, 8, 9, 10, 11, 12, 13, 14, 15, 16, 17, 18, 19] for further details):

$$
\boldsymbol{X}=\sum_{k=1}^{+\infty} \sqrt{\lambda_{k}} \boldsymbol{u}^{k} \eta_{k},
$$

where couples $\left(\boldsymbol{u}_{k}, \lambda_{k}\right)$ are solution of the Fredholm eigenvalue problem, such that for all $\left(s, s^{\prime}\right) \in \Omega \times \Omega$ :

$$
\begin{gathered}
E\left[\boldsymbol{X}(s) \boldsymbol{X}\left(s^{\prime}\right)^{T}\right] \approx\left[R_{\boldsymbol{X} \boldsymbol{X}}\left(s, s^{\prime}\right)\right]=\frac{1}{\nu^{\exp }} \sum_{i=1}^{\nu^{\exp }} \boldsymbol{x}^{i}(s)\left(\boldsymbol{x}^{i}\left(s^{\prime}\right)\right)^{T}, \\
\int_{\Omega}\left[R_{\boldsymbol{X} \boldsymbol{X}}\left(s, s^{\prime}\right)\right] \boldsymbol{u}^{k}(s) d s=\lambda_{k} \boldsymbol{u}^{k}\left(s^{\prime}\right), \\
\int_{\Omega} \boldsymbol{u}^{k}(s) \boldsymbol{u}^{\ell}(s) d s=\delta_{k \ell}, \quad \lambda_{1} \geq \lambda_{2} \geq \ldots \rightarrow 0,
\end{gathered}
$$

where $\delta_{k \ell}$ is the Kronecker symbol, equal to one if $k=\ell$ and zero otherwise, and $\left\{\eta_{1}, \eta_{2}, \ldots\right\}$ are uncorrelated but a priori dependent random variables that verify by construction the following equalities:

$$
E\left[\eta_{k} \eta_{\ell}\right]=\delta_{k \ell}
$$

As an illustration, a particular projection of the empirical estimation of the covariance matrixvalued function of $\boldsymbol{X},\left[R_{\boldsymbol{X} \boldsymbol{X}}\right]$, is represented in Figures 1, whereas four particular eigenfunctions, $\boldsymbol{u}^{1}, \boldsymbol{u}^{5}, \boldsymbol{u}^{10}$ and $\boldsymbol{u}^{25}$, are shown in Figure 2]. It can be noticed that the vectorial approach allows us to take into account the spatial dependencies between the different track irregularities.

From a practical point of view, this sum is truncated to its $N_{\eta}$ most influential terms:

$$
\boldsymbol{X} \approx \boldsymbol{X}^{\left(N_{\eta}\right)}=\sum_{k=1}^{N_{\eta}} \sqrt{\lambda_{k}} \boldsymbol{u}^{k} \eta_{k}
$$



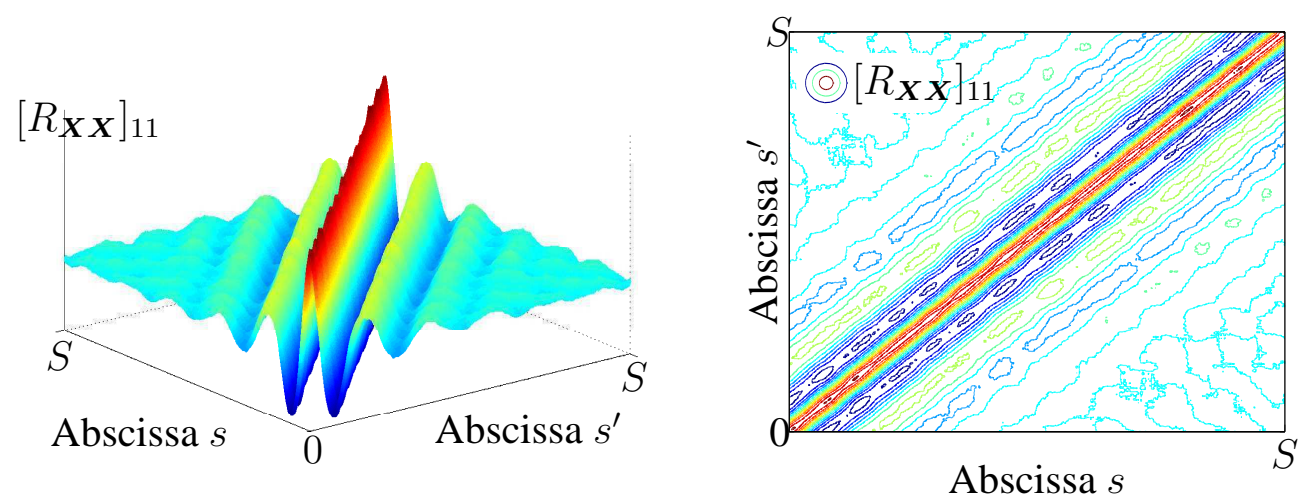

Figure 1: Graphs of $\left[R_{\boldsymbol{X} \boldsymbol{X}}\right]_{11}$.
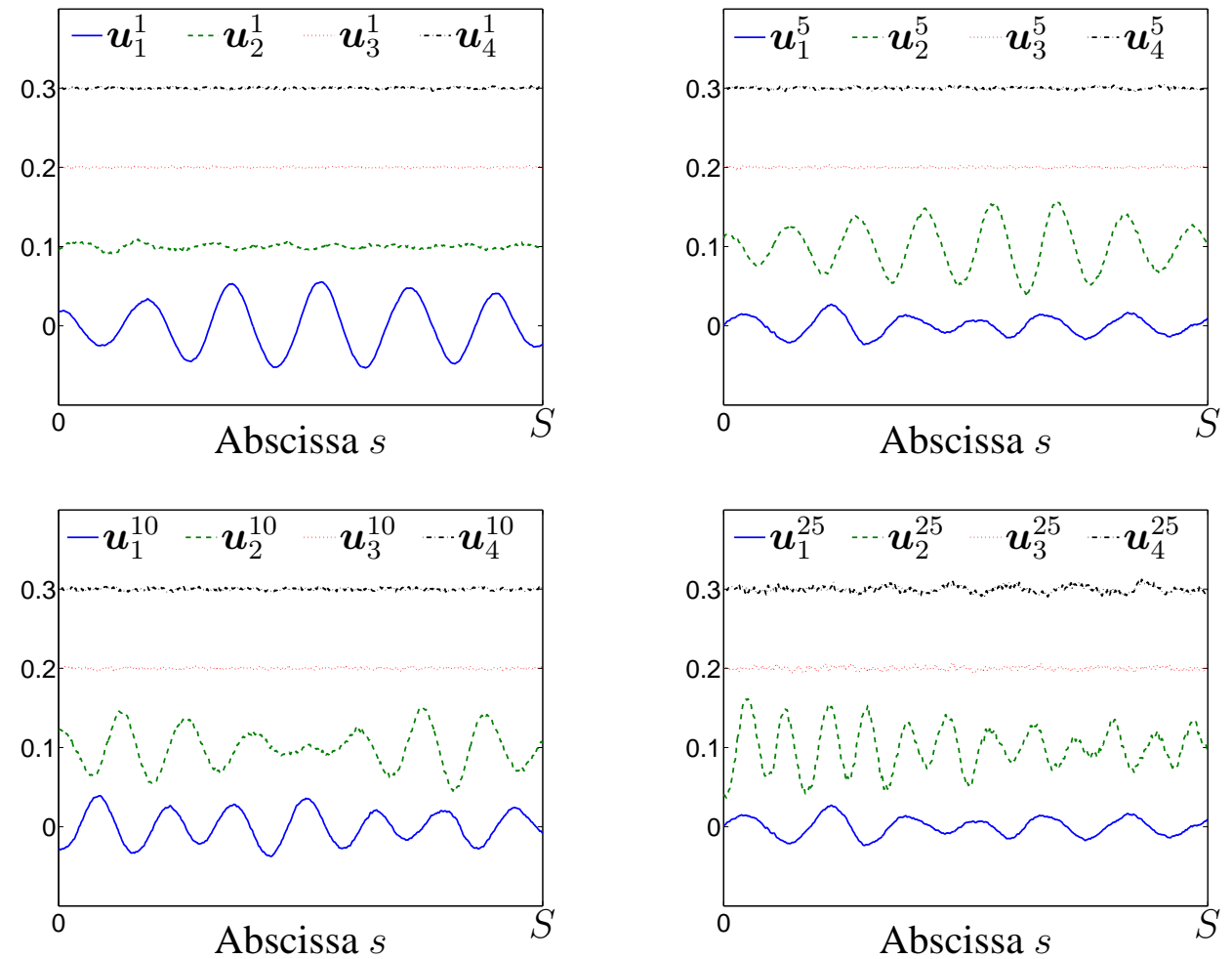

Figure 2: Graphs of four particular eigenvectors $\boldsymbol{u}^{1}, \boldsymbol{u}^{5}, \boldsymbol{u}^{10}$ and $\boldsymbol{u}^{25}$ $\left(\times 10^{-3} S\right)$. 
where the amplitude of the truncation residue, $\boldsymbol{X}-\boldsymbol{X}^{\left(N_{\eta}\right)}$, is evaluated by the normalized $L_{2}$ error, $\varepsilon_{K L}^{2}\left(N_{\eta}\right)$, such that:

$$
\varepsilon_{K L}^{2}\left(N_{\eta}\right)=\frac{\left\|\boldsymbol{X}-\boldsymbol{X}^{\left(N_{\eta}\right)}\right\|_{2}^{2}}{\|\boldsymbol{X}\|_{2}^{2}}=1-\frac{\sum_{k \leq N_{\eta}} \lambda_{k}}{\|\boldsymbol{X}\|_{2}^{2}},
$$

where for all second order and mean-square continuous vector-valued random field $Z$,

$$
\|\boldsymbol{Z}\|_{2}^{2}=E\left[\int_{\Omega} \int_{\Omega} \boldsymbol{Z}(s)^{T} \boldsymbol{Z}\left(s^{\prime}\right) d s d s^{\prime}\right] .
$$

The higher $N_{\eta}$, the more precise the characterization of the track geometry, but the more difficult the characterization of the random vector $\boldsymbol{\eta}=\left(\eta_{1}, \ldots, \eta_{N_{\eta}}\right)$. As a good compromise, the truncation parameter $N_{\eta}$ is fixed to the value 940 in the following, which corresponds to an error threshold of $1 \%$ for $\varepsilon_{K L}^{2}$.

The second step of the modeling of $\boldsymbol{X}$ is the characterization in inverse of the multidimensional probability density function (PDF) of $\boldsymbol{\eta}, p_{\boldsymbol{\eta}}$. In this prospect, a polynomial chaos expansion (PCE) method (see [20, 21, 22, 23, 24, 25, 26, 27, 28, 29, 30] for further details about the PCE identification in inverse) is used, which corresponds to a direct projection of $\boldsymbol{\eta}$ on a chosen polynomial hilbertian basis $\mathcal{B}_{\text {orth }}=\left\{\psi_{j}(\boldsymbol{\xi}), 1 \leq j\right\}$ of all the second-order random vectors with values in $\mathbb{R}^{N_{\eta}}$, such that:

$$
\boldsymbol{\eta}=\sum_{j=1}^{+\infty} \boldsymbol{y}^{(j)} \psi_{j}(\boldsymbol{\xi})
$$

This sum is once again truncated with respect to two truncation parameters, $N$ and $N_{g}$, such that:

$$
\boldsymbol{\eta} \approx \boldsymbol{\eta}^{\text {chaos }}(N)=\sum_{j=1}^{N} \boldsymbol{y}^{(j)} \psi_{j}\left(\xi_{1}, \ldots, \xi_{N_{g}}\right),
$$

where projection basis $\left\{\psi_{1}\left(\xi_{1}, \ldots, \xi_{N_{g}}\right), \cdots, \psi_{N}\left(\xi_{1}, \ldots, \xi_{N_{g}}\right)\right\}$ is now defined as the set gathering the $N$ polynomial functions of total degree inferior to $p$, which are normalized with respect to the PDF $p_{\xi_{1}, \ldots, \xi_{N_{g}}}$ of $\left(\xi_{1}, \ldots, \xi_{N_{g}}\right)$ :

$$
\begin{gathered}
\psi_{j}\left(\xi_{1}, \ldots, \xi_{N_{g}}\right)=\sum_{q=1}^{N} c_{q j} \xi_{1}^{\alpha_{1}^{(q)}} \times \cdots \times \xi_{N_{g}}^{\alpha_{N_{g}}^{(q)}}, \quad \sum_{\ell=1}^{N_{g}} \alpha_{\ell}^{(q)} \leq p, \\
\int_{\mathbb{R}^{N_{g}}} \psi_{j}(\boldsymbol{x}) \psi_{n}(\boldsymbol{x}) p_{\xi_{1}, \ldots, \xi_{N_{g}}}(\boldsymbol{x}) d \boldsymbol{x}=\delta_{j n} .
\end{gathered}
$$

For given values of $N$ and $N_{g}$, identifying the distribution of $\boldsymbol{\eta}^{\text {chaos }}(N)$ amounts therefore to identifying the values of the PCE projection coefficients, $\left\{\boldsymbol{y}^{(j)}, 1 \leq j \leq N\right\}$, from the available information about $\boldsymbol{\eta}$. According to Eqs. (11), (14) and (15), this available information corresponds to the $\nu^{\text {exp }}$ independent realizations of $\boldsymbol{\eta}^{\text {chaos }}(N),\left\{\boldsymbol{\eta}^{1}, \cdots, \boldsymbol{\eta}^{\nu^{\exp }}\right\}$, which can be deduced from the $\nu^{\exp }$ independent realizations of $\boldsymbol{X},\left\{\boldsymbol{x}^{1}, \cdots, \boldsymbol{x}^{\nu^{\mathrm{exp}}}\right\}$, such that:

$$
\eta_{k}^{i}=\frac{1}{\sqrt{\lambda_{k}}} \int_{\Omega}\left(\boldsymbol{x}^{i}(s)\right)^{T} \boldsymbol{u}^{k}(s) d s, \quad 1 \leq i \leq \nu^{\exp }, \quad 1 \leq k \leq N_{\eta}
$$




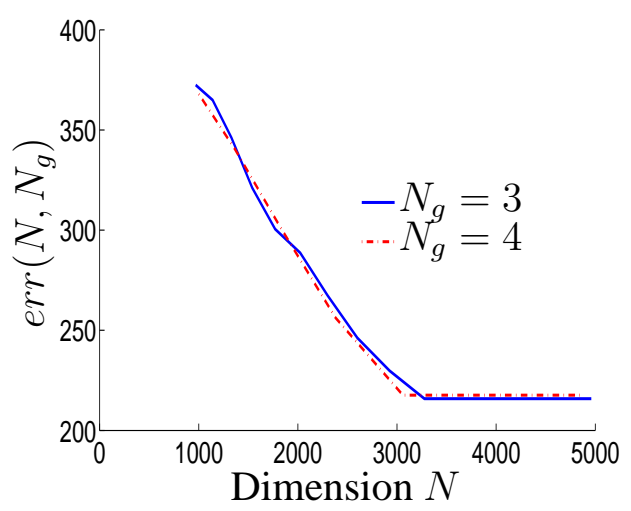

Figure 3: Convergence analysis for the PCE expansion of $\boldsymbol{\eta}$.

In [28, 29], it has been shown that a good approach to identify such coefficients is to search them as the arguments that maximize the likelihood of random vector $\boldsymbol{\eta}^{\text {chaos }}(N)$ at the experimental points $\left\{\boldsymbol{\eta}^{1}, \cdots, \boldsymbol{\eta}^{\nu^{\exp }}\right\}$.

Finally, the last step of the identification of the distribution of $\boldsymbol{\eta}^{\text {chaos }}(N)$ is the justification of the values for the truncation parameters $N$ and $N_{g}$. In this prospect, the log-error function $\operatorname{err}\left(N, N_{g}\right)$ is introduced to quantify the amplitude of the residue of the PCE truncation, $\boldsymbol{\eta}-$ $\boldsymbol{\eta}^{\text {chaos }}(N)$, such that:

$$
\begin{gathered}
\operatorname{err}\left(N, N_{g}\right)=\sum_{k=1}^{N_{\eta}} \operatorname{err}_{k}\left(N, N_{g}\right), \\
\operatorname{err}_{k}\left(N, N_{g}\right)=\int_{\mathrm{BI}_{k}}\left|\log _{10}\left(p_{\eta_{k}}\left(x_{k}\right)\right)-\log _{10}\left(p_{\eta_{k}^{\text {chaos }}}\left(x_{k}\right)\right)\right| d x_{k},
\end{gathered}
$$

where $\mathrm{BI}_{k}$ is the domain bounding the experimental values of $\eta_{k}, p_{\eta_{k}}$ and $p_{\eta_{k}^{\text {chaos }}}$ are the PDFs of $\eta_{k}$ and $\eta_{k}^{\text {chaos }}(N)$ respectively. Truncation parameters $N$ and $N_{g}$ can thus be chosen with respect to a given error threshold for $\operatorname{err}\left(N, N_{g}\right)$.

For our study, $\boldsymbol{\xi}$ is a $N_{g}$-dimension random vector, whose components are independent and uniformly distributed between -1 and 1 . According to Figure 3, which represents the convergence of error function $\operatorname{err}\left(N, N_{g}\right)$ with respect to $N$ and $N_{g}$, truncation parameters $N$ and $N_{g}$ are chosen equal to 3,276 and 3 respectively.

To conclude, once truncation parameters $N_{\eta}, N, N_{g}$ have been identified according to convergence analysis, once PCE projection coefficients $\left\{\boldsymbol{y}^{(j)}, 1 \leq j \leq N\right\}$ have been computed with the advanced algorithms described in [29] and [28], the track irregularity random field is completely characterized and can finally be estimated as:

$$
\boldsymbol{X} \approx \sum_{k=1}^{N_{\eta}} \sqrt{\lambda_{k}} \boldsymbol{u}^{k} \sum_{j=1}^{N} y_{k}^{(j)} \psi_{j}\left(\xi_{1}, \ldots, \xi_{N_{g}}\right) .
$$

For each realization of random vector $\left(\xi_{1}, \ldots, \xi_{N_{g}}\right)$, a representative and realistic track geometry of length $S$ can finally be generated. 


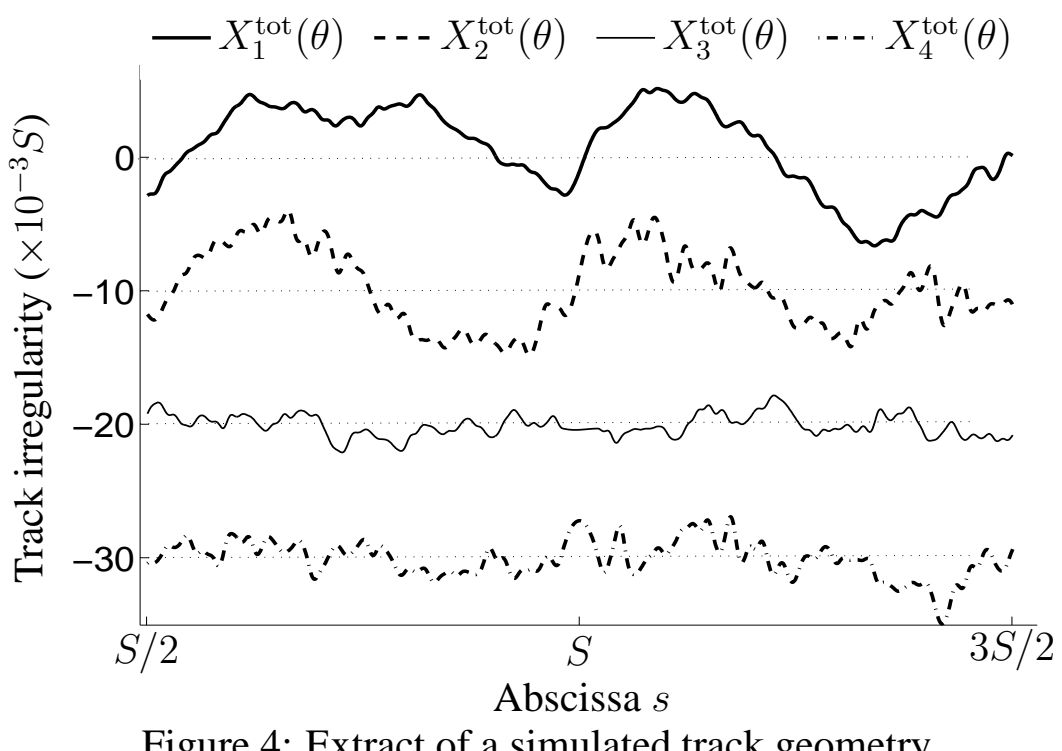

Figure 4: Extract of a simulated track geometry.

\subsubsection{Definition of the global model}

According to Section 2 and to the local-global hypothesis, a realistic track geometry of length $S^{\text {tot }}=N_{\mathcal{T}} S$ can be generated from the concatenation of $N_{\mathcal{T}}$ track geometries of same length $S$. However, a particular attention has to be paid to the interface between two different realizations of $\boldsymbol{X}$. Indeed, these junctions have to guarantee the continuity of the track irregularity vector and at least the continuity of its first and second order spatial derivatives in order to avoid an artificial perturbation of the train dynamics. Spline interpolations on a length corresponding to the minimal wavelength of the measured irregularities are then used to fulfill these continuity conditions.

From the local stochastic modeling developed in Section 3.2.1, it is now possible to generate track geometries of length $S^{\text {tot }}$, which are representative of the whole track geometry of the measured high speed line. As an illustration, an extract of length $S$ of a complete generated track geometry $\boldsymbol{X}^{\text {tot }}(\theta)$ is represented in Figure 4, This graph is centered at a junction between the two first realizations that stem from the local stochastic modeling of $\boldsymbol{X}$. The values of the four irregularity fields, for which mean value is zero, have been translated on purpose to allow a better visualization of the results.

\section{STEP C: PROPAGATION OF THE TRACK VARIABILITY TO THE TRAIN RE- SPONSE}

Sections 2 and 3 have presented the railway stochastic modeling and the characterization of the input variability. This variability has now to be propagated through the model. After presenting the chosen method for this propagation, this Section describes two applications of the whole method. The first one analyses the influence of an increase of the train speed on the three criteria of interest. The second one underlines in what extent such methods can be used to compare the dynamical response of different high speed trains. 


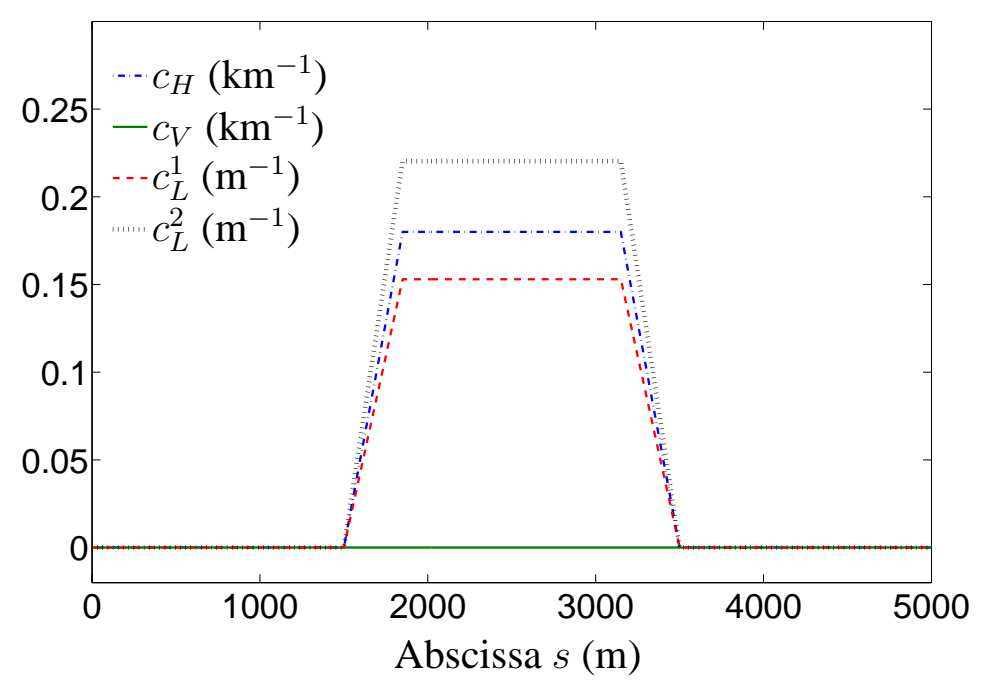

Figure 5: Track Design of the simulated railway track.

\subsection{Monte Carlo simulation}

From Section 2, three outputs of the railway simulation are studied in this work: a shifting criterion, $C_{1}=\left(Y_{\ell}+Y_{r}\right)_{\max }$, a derailment criterion, $C_{2}=(Y / Q)_{\max }$, and a wear criterion, $C_{3}=(T \gamma)$.

As the relation between the three criteria and the track irregularity random field, $\boldsymbol{X}$, is very complex and strongly non-linear, the Monte Carlo method is a good approach to characterize the statitical properties of $C_{1}, C_{2}$ and $C_{3}$, as the convergence of these properties is independent of the statistical dimension, $N_{\eta}$, of the KL approximation of $\boldsymbol{X}$ that is very high.

In this prospect, a measured track design of length $5 \mathrm{~km}$ around a high speed line horizontal curve is considered. The track superelevations $c_{L}^{1}$ and $c_{L}^{2}$ have been introduced to compensate the inertial acceleration in curve for a train that runs at speeds $\mathcal{S}$ and $1.2 \mathcal{S}$ respectively. For confidentiality reasons, the value of $\mathcal{S}$ is not given in this paper. Then, $\nu=500$ independent track irregularities, $\left\{\boldsymbol{X}^{\text {tot }}\left(\theta_{1}\right), \ldots, \boldsymbol{X}^{\text {tot }}\left(\theta_{\nu}\right)\right\}$, of total length $5 k m$ are generated thanks to the local-global approach and to the stochastic modeling of random field $\boldsymbol{X}$ on a length $S$, such that $\nu=500$ realistic and representative running conditions around the same curve are available.

Coupled to the model of a train, these geometries can now be used in any rigid-multibodies railway software to characterize its dynamic behavior. For our study, a commercial code, which is called Vampire, has been used.

\subsection{Influence of an increase of the speed on the quantities of interest}

The first application of the whole method deals with the influence of the speed on the distributions of the three criteria considered for a normalized high speed train. Railway simulations are therefore performed on the same $\nu$ realistic and representative track geometries, at the four speeds $\mathcal{S} 1=\mathcal{S}, \mathcal{S} 2=1.1 \mathcal{S}, \mathcal{S} 3=1.2 \mathcal{S}$ and $\mathcal{S} 4=1.3 \mathcal{S}$. Two other sets of simulations have then been carried out for a different value of the track superelevation at speeds $\mathcal{S} 3=1.2 \mathcal{S}$ and $\mathcal{S} 4=1.3 \mathcal{S}$ in order to quantify the importance of this track design parameter with respect to the three criteria studied.

For each speed, the PDFs of each criterion $C_{1}, C_{2}$ and $C_{3}$ are then estimated using kernel smoothing on the $\nu=500$ independent railway simulations. These PDFs are represented in 
Figure 7. In this figure, the non-linearity of the system can be noticed, as the consequences of an increase of the speed of $10 \%$ to $30 \%$ are much higher than $30 \%$ for each criterion considered. In particular, an increase of $30 \%$ of the speed of the train can lead to an increase of more than $500 \%$ of the contact wear if the track superelevation is not adjusted. In addition, this figure emphasizes the importance of the adjustment of the track superelevation to the speed, in terms of minization of the wear, of the shifting risk and of the risk of derailment.

\subsection{Comparison of three high speed trains}

In this section, it is supposed that three different models of three concurrential high speed trains, $\mathcal{V} 1, \mathcal{V} 2$ and $\mathcal{V} 3$ are available, for which parameters have been carefully identified from experimental measurements. Therefore, the method proposed in this paper allows us to compare the dynamical response of these three trains when they are excited at speed $\mathcal{S}$, by a representative set of the variable track conditions they can be confronted to during their lifecycle. The results of this analysis are shown in Figure 7 In particular, criteria $C_{1}$ and $C_{3}$ could be interessant indicators to compare the agressiveness of each train, whereas criterion $C_{2}$ could be used to quantify the global stability in curve of each train.

\section{CONCLUSIONS}

A method to propagate the track geometry variability through railway mechanical simulations is nowadays of great interest to face always more challenging railway issues. In this prospect, this paper has presented a general method to completely parametrize the track geometry and its variability. This method is based on a local-global approach, and a double projection, which can be applied to many other mechanical systems. First, a Karhunen-Loeve expansion is used to decompose the projection of the random field as a truncated weighted sum of deterministic spatial functions, for which weights are a priori dependent but uncorrelated random variables. The distribution of the high dimension random vector that gathers all these weights is then characterized thanks to a truncated PCE. At last, complete track geometries that are realistic and representative of a whole railway network can be generated. These geometries can finally be used in any railway software to characterize the dynamic behavior of trains. To this end, two applications of the whole method have been described in this paper. The first one analyses the impact of an increase of the speed on the train stability, whereas the second one shows in what extent such an approach could be used to compare competitive high speed trains with respect to their response on a set of representative track conditions.

\section{REFERENCES}

[1] C. Allery, A. Hambouni, D. Ryckelynck, and N. Verdon. A priori reduction method for solving the two-dimensional burgers' equations. Applied Mathematics and Computation, 217:6671-6679, 2011.

[2] J.A. Atwell and B.B. King. Proper orthogonal decomposition for reduced basis feedback controllers for parabolic equations. Math. Comput. Modell., 33 (1-3):1-19, 2001.

[3] G. Berkooz, P. Holmes, and J.L. Lumley. The proper orthogonal decomposition in the analysis of turbulent flows. Annu. Rev. Fluid Mech., 25:539-575, 1993. 

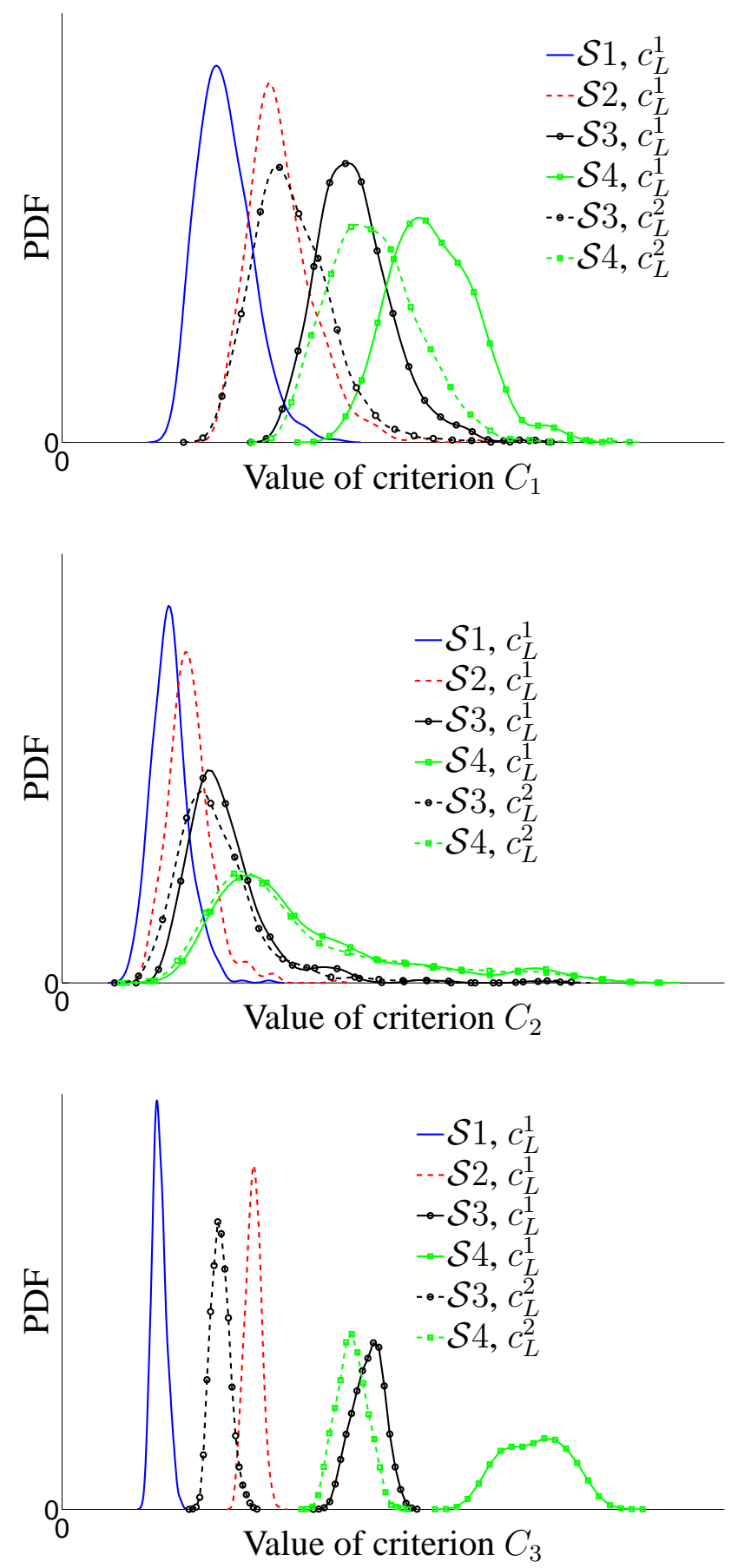

Figure 6: Representation of the evolutions of the PDFs of the three criteria considered with respect to an increase of the train speed. 

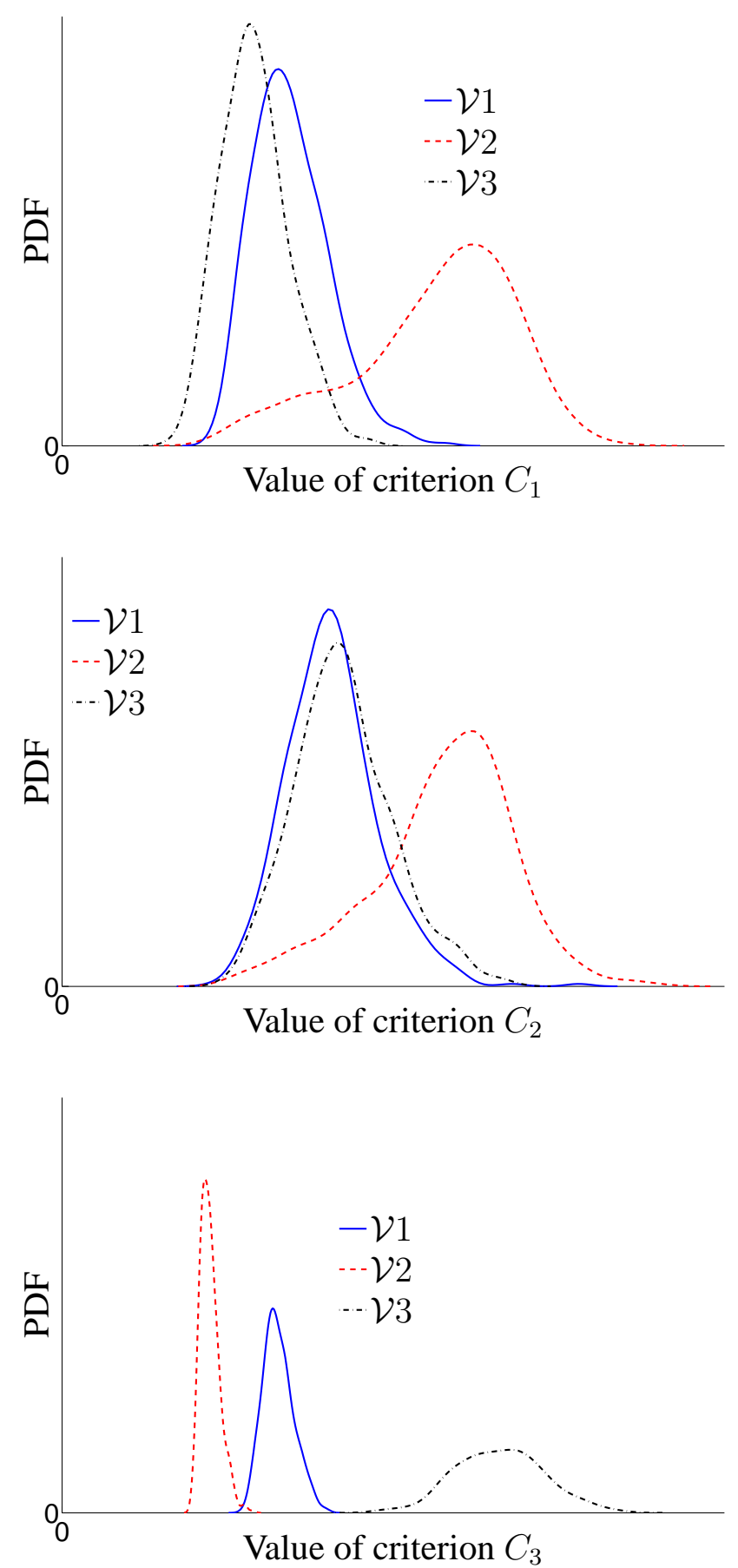

Figure 7: Comparison between the PDFs of the three criteria considered with respect to the train characteristics. 
[4] G. P. Brooks and J. M. Powers. A karhunen-loève least-squares technique for optimization of geometry of a blunt body in supersonic flow. Journal of Computational Physics, 195:387-412, 2004.

[5] E.A. Christensen, M. Brons, and J.M. Sorensen. Evaluation of proper orthogonal decomposition-based decomposition techniques applied to parameter dependent nonturbulent flows. SIAM J. Sci. Comput, 21 (4):1419-1434, 2000.

[6] S.P. Huang, S.T. Quek, and K.K. Phoon. Convergence study of the truncated karhunenloève expansion for simulation of stochastic processes. Int J Num Meth Engng, 52 (9):1029-43, 2001.

[7] K. Kunisch and S. Volkwein. Galerkin proper orthogonal decomposition methods for parabolic problems. Numer. Math., 90 (1):117-148, 2001.

[8] L. Li, K. Phoon, and S. Quek. Comparison between karhunen-loève expansion and translation-based simulation of non-gaussian processes. Computers and Structures, 85:264-76, 2007.

[9] X. Ma and N. Zabaras. Kernel principal component analysis for stochastic input model generation. Comptes rendus de l'Académie des sciences de Paris, 220, 1945.

[10] Y. M. Marzouk and H. N. Najm. Dimensionality reduction and polynomial chaos acceleration of bayesian inference in inverse problems. J. Comput. Phys., 228:1862-1902, 2009.

[11] A. Nouy and O.P. Le Maître. Generalized spectral decomposition method for stochastic non-linear problems. J. Comput. Phys., 228 (1):202-235, 2009.

[12] K.K. Phoon, S.P. Huang, and S.T. Quek. Implementation of karhunen-loeve expansion for simulation using a wavelet-galerkin scheme. Probabilistic Engineering Mechanics, 17:293-303, 2002.

[13] K.K. Phoon, S.P. Huang, and S.T. Quek. Simulation of strongly non-gaussian processes using karhunen-loeve expansion. Probabilistic Engineering Mechanics, 20:188$198,2005$.

[14] Christoph Schwab and Radu Alexandru Todor. Karhunen-loeve approximation of random fields by generalized fast multipole methods. Journal of Computational Physics, 217:100$122,2006$.

[15] P.D. Spanos and B.A. Zeldin. Galerkin sampling method for stochastic mechanics problems. Journal of Engineering Mechanics, 120 (5):1091-1106, 1994.

[16] P.D. Spanos, M. Beer, and J. Red-Horse. Karhunen -loève expansion of stochastic processes with a modified exponential covariance kernel. Journal of Engineering Mechanics, 133 (7):773-779, 2007.

[17] B. Wen and N. Zabaras. A multiscale approach for model reduction of random microstructures. Computational Materials Science, 63:269-285, 2012. 
[18] M.M.R. Williams. The eigenfunctions of the karhunen-loeve integral equation for a spherical system. Propabilistic Engineering Mechanics, 26:202-207, 2011.

[19] S. Q. Wu and S. S. Law. Statistical moving load identification including uncertainty. Probabilistic Engineering Mechanics, 29:70-78, 2012.

[20] R. Ghanem and P.D. Spanos. Polynomial chaos in stochastic finite elements. Journal of Applied Mechanics, Transactions of teh ASME 57:197-202, 1990.

[21] R. Ghanem and P. D. Spanos. Stochastic Finite Elements: A Spectral Approach, rev. ed. Dover Publications, New York, 2003.

[22] M. Arnst, R. Ghanem, and C. Soize. Identification of bayesian posteriors for coefficients of chaos expansions. Journal of Computational Physics, 229 (9):3134-3154, 2010.

[23] S. Das, R. Ghanem, and S. Finette. Polynomial chaos representation of spatio-temporal random field from experimental measurements. J. Comput. Phys., 228:8726-8751, 2009.

[24] C. Desceliers, R. Ghanem, and C. Soize. Maximum likelihood estimation of stochastic chaos representations from experimental data. Internat. J. Numer. Methods Engrg., 66:978-1001, 2006.

[25] C. Desceliers, C. Soize, and R. Ghanem. Identification of chaos representations of elastic properties of random media using experimental vibration tests. Comput. Mech., 39:831838, 2007.

[26] R.G. Ghanem and A. Doostan. On the construction and analysis of stochastic models: Characterization and propagation of the errors associated with limited data. J. Comput. Phys., 217:63-81, 2006.

[27] Y. M. Marzouk, H. N. Najm, and L. A. Rahn. spectral methods for efficient bayesian solution of inverse problems. J. Comput. Phys., 224:560-586, 2007.

[28] G. Perrin, C. Soize, D. Duhamel, and C. Funfschilling. Identification of polynomial chaos representations in high dimension from a set of realizations. SIAM J. Sci. Comput., 34(6):2917-2945, 2012.

[29] C. Soize. Identification of high-dimension polynomial chaos expansions with random coefficients for non-gausian tensor-valued random fields using partial and limited experimental data. Computer Methods in Applied Mechanics and Engineering, 199:2150-2164, 2010 .

[30] C. Soize. Generalized probabilistic approach of uncertainties in computational dynamics using random matrices and polynomial chaos decompositions. Internat. J. Numer. Methods Engrg., 81:939-970, 2010. 